\title{
Pyruvate Kinase Inhibitor TLN-232
}

National Cancer Institute

\section{Source}

National Cancer Institute. Pyruvate Kinase Inhibitor TLN-232. NCI Thesaurus. Code C67087.

A synthetic cyclic heptapeptide with potential antineoplastic activity. Pyruvate kinase (PK) inhibitor TLN-232 targets pyruvate kinase M2 (M2PK), which may disrupt tumor cell anaerobic glycolysis. M2PK is a dimeric isoform of PK and the predominant PK isoform found in tumor cells 